\title{
A pilot and comparative study between pathological and serological levels of immunoglobulin and complement among three kinds of primary glomerulonephritis
}

Jin Dong ${ }^{1 \dagger}$, Tianhao Peng ${ }^{2+}$, Jing Gao ${ }^{1}$, Xingwang Jia ${ }^{1}$, Guangtao Yan ${ }^{1}$ and Yong Wang ${ }^{3^{*}}$ (D)

\begin{abstract}
Background: Immunoglobulin A nephropathy (IgAN), membranous nephropathy (MN) and minimal-change disease (MCD) are three common types of glomerulonephritis in China. Pathological diagnosis based on renal biopsy is the criterion and the golden standard for diagnosing the sub-types of primary or secondary glomerulonephritis. Immunoglobulin and complements might be used in the differential diagnosis of glomerulonephritis without renal biopsies. However, the relationship between IF intensities of immune proteins and the corresponding serum levels remained unclear, and seldom studies combine histopathological examination results and blood tests together for a predictive purpose. This study was considered as a pilot study for integrating histopathological indicators into serum parameters for exploring the relationship of IF intensity and serum values of immunoglobulin and complement, and for screening and investigating effective indicators inlgAN, MN and MCD.

Methods: Renal tissue immunofluorescence (IF) intensity grades and serum levels of immunoglobulin and complements (IgG, IgA, IgM, C3 and C4) were retrospectively analyzed in 236 cases with IgAN, MN or MCD. IF grades were grouped as negative $(-)$, positive $(+)$ or strong positive $(++)$ with both high and low magnification of microscope. Other serum indicators such as urea nitrogen (BUN), creatinine (Crea) and estimated glomerular filtration rate (eGFR) were also evaluated among the groups.

(Continued on next page)
\end{abstract}

\footnotetext{
*Correspondence: wangyong301@263.net

${ }^{\dagger}$ Jin Dong and Tianhao Peng contributed equally to this work.

${ }^{3}$ Department of Nephrology, Chinese PLA Institute of Nephrology, State Key Laboratory of Kidney Diseases, National Clinical Research Center for Kidney Diseases, Medical Institution Conducting Clinical Trials, Chinese PLA General Hospital, Beijing, China

Full list of author information is available at the end of the article
}

(c) The Author(s). 2018 Open Access This article is distributed under the terms of the Creative Commons Attribution 4.0 International License (http://creativecommons.org/licenses/by/4.0/), which permits unrestricted use, distribution, and reproduction in any medium, provided you give appropriate credit to the original author(s) and the source, provide a link to the Creative Commons license, and indicate if changes were made. The Creative Commons Public Domain Dedication waiver (http://creativecommons.org/publicdomain/zero/1.0/) applies to the data made available in this article, unless otherwise stated. 
(Continued from previous page)

Results: There were difference in IgA, IgG and C3 IF intensity grades among IgAN, MN and MCD groups ( $p=9.82 \mathrm{E}-43$, 4.60E-39, 7.45E-15, respectively). Serum values of BUN, Crea, eGFR, IgG, IgA, IgM and C4 showed difference in three groups (BUN: $p=0.045$, Crea: $p=3.45 \mathrm{E}-5, \mathrm{eGFR}: p=0.005$, IgG: $p=1.68 \mathrm{E}-14, \operatorname{lgA}: p=9.14 \mathrm{E}-9$, IgM: $p=0.014,(4: p=0.026$ ). eGFR had the trend to decrease with enhanced IgA IF positive grades ( $p=8.99 \mathrm{E}-4)$; Crea had trends to decrease with both enhanced IgA and IgG IF intensity grades ( $p=2.06 \mathrm{E}-6,2.94 \mathrm{E}-5$, respectively). In all subjects, serum IgA levels was inversely correlated with eGFR( $r=-0.216, p=0.001)$ and correlated with Crea levels $(r=0.189, p=0.004)$; serum IgG and Crea showed no correlation which were discordance with inverse correlation of IgG IF grade and Crea $(r=0.058, p=0.379)$. lgG serum level was inverse correlated with its IF grades ( $p=3.54 \mathrm{E}-5, p=7.08 \mathrm{E}-6$, respectively); C3 serum levels had significantly difference between Neg and positive $(+)$ group $(p=0.0003)$. IgA serum level was positive correlated with its IF grades (Neg-(+): $p=0.0001 ;(+)-(++): p=0.022 ; \mathrm{Neg}-(++): p=2.01 \mathrm{E}-10)$. After matching comparison among C3 groups, C3 Neg. group and C3 ++ group had difference ( $\left.{ }^{*} p=0.017\right)$. C4 had all negative IF expression in all pathological groups. In IgAN subjects, there were statistical differences of serum C3 levels between its pathological Neg and positive (+) group $(p=0.026)$, and serum IgA levels showed difference between IgA pathological positive $(+)$ and $(++)(p=0.007)$. In $M N$ subjects, slgG levels showed difference between IgG pathological IF grade positive $(+)$ and $(++)(p=0.044)$; serum C3 levels showed difference between C3 pathological IF grade Neg and positive $(+)(p=0.005)$; and serum IgA levels showed difference between Neg and positive $(+)(p=0.040)$. In IgAN, eGFR showed serum IgA levels had significant differences among groups $(p=0.007)$ and had increasing trend with enhanced its IF grades $\left(P_{\text {trend }}=0.016\right)$. There were also difference between IgG group Neg and positive $(+)\left(p=0.005, P_{\text {trend }}=0.007\right)$ in IgAN. In MN, serum IgG levels had significant differences among IF groups $(p=0.034)$ and had decreasing trend with its enhanced IF grades $\left(P_{\text {trend }}=0.014\right)$. Serum $C 3$ concentrations also were found distinctive among IF groups $(p=0.016)$ and had in inverse correlation with its enhanced IF grades $\left(P_{\text {trend }}=0.004\right)$.

Discussion: Our research cross contrasts several immunoprotein IF intensities and relevant serum levels in three kinds of primary glomerular nephritis, and finally acquired helpful results for understanding the relationships between pathological presentation and serological presentation of immunoproteins in kidney diseases. Furthermore, this pilot study is offering a possible method for the analysis of combination of pathology and serology.

Conclusion: Different pathological types of nephritis presented different expression patterns of immunoglobulin and complement, especially IgA and IgG, which suggested different pathogenesis involved in the development of IgAN and MN. Furthermore, either in tissue or in serum, increased IgA level was closely related with renal function in all of the patients.

Keywords: Immunoglobulin A nephropathy, Membranous nephropathy, Minimal change disease, Immunoglobulin, Complement

\section{Background}

Immunoglobulin A nephropathy (IgAN), membranous nephropathy $(\mathrm{MN})$ and minimal-change disease (MCD) are three common types of glomerulonephritis in China $[1,2]$. So far, pathological diagnosis based on invasive renal biopsy is the criterion and the golden standard for diagnosing the sub-types of primary or secondary glomerulonephritis, which is defined by the types of dominantly accumulated and infiltrated immunoglobulin in kidney tissue, such as IgAN is known as IgA or IgA-dominant immune complex deposition in glomerular mesangial through immunofluorescence. The formation and the deposition of immunoglobulin complexes play a key role in the pathogenesis of glomerulonephritis. An animal study on the progressive of glomerulonephropathy revealed that the formation of IgM deposition was earlier than of IgA and IgG deposition, and therefore IgA and IgG might be related with the progression of glomerular lesions [3]. Zhang et al. [4] found IgA/C3 changed with the progression of IgAN, whereas a study conducted by Yang et al. [5] showed decreased serum C3 level was not a leading factor in renal progression in IgAN. Mizerska-Wasiak $M$ et al. [6] found that the IgA/C3 ratio was a useful marker for evaluating severity of lesions by Oxford classification in children with IgAN. All of those researches indicated immunoglobulin and complements might be used in the differential diagnosis of glomerulonephritis without renal biopsies. The evidence showed serum IgA value, serum IgA/C3 ratio and serum IgG value were related with IgAN or $\mathrm{MN}$, so we supposed that blood markers might imitate histopathological changes and played a predictive role in diagnosis of the diseases. However, pathological parameters and sera parameters were always kept apart analysis in the most studies. The relationship between IF intensities of immune proteins and the corresponding serum levels remained unclear, and seldom studies combine histopathological examination results and blood tests together for a predictive purpose. 
This study was considered as a pilot study for integrating histopathological indicators into serum parameters for exploring the relationship of IF intensity and serum values of immunoglobulin and complement, and for screening and investigating effective indicators for the diseases.

\section{Methods}

Subjects, inclusion and exclsion criteria and parameters This study was a retrospective cohort study and was approved by the Medical Ethics Committee of the Chinese PLA general hospital. All patients had signed on the patient consents form for their agreements.

Patient's inclusion criteria and exclusion criteria were described in Gao J's study [7].

Data of two hundred and thirty-six patients who were diagnosed as IgAN, MN or MCD in Chinese PLA general hospital from Jan 1st, 2015 to Jan 1st, 2016 were retrospectively analyzed. The include parameters were as follows: IF intensity grades of immunoglobulin and complements (including IgG, IgM, IgA, C3, C4) with renal biopsies; serum levels of IgG, IgM, IgA, C3, C4, urea nitrogen (BUN) and creatinine (Crea); estimated glomerular filtration rate (eGFR) were also collected.

\section{Methods}

IF intensity grades of immunoglobulin and complement (IgG, IgM, IgA, C3, C4) were evaluated immunofluorescence of renal tissue, as a typical method of immunohistochemistry [8-10]. [As shown in Fig. 1, the classification of negative(Neg.), positive(+) and strong positive $(++)$ was as follows: with immunofluorescence(high magnification:X200; low magnification: X10), negative(Neg.) is defined as: no fluorescence was observed in glomerular; positive $(+)$ is defined as: fluorescence was observed by microscope with high magnification:X200, and fluorescence is faintly visible by microscope with low magnification: $\mathrm{X} 10$; positive $(++)$ is defined as: fluorescence was clearly visible by microscope with high magnification:X200, and fluorescence is visible by microscope with low magnification: X10).]

Serum IgG, IgM, IgA, C3, C4 levels were tested on BNII analyzer (Siemens). Serum BUN and Crea were

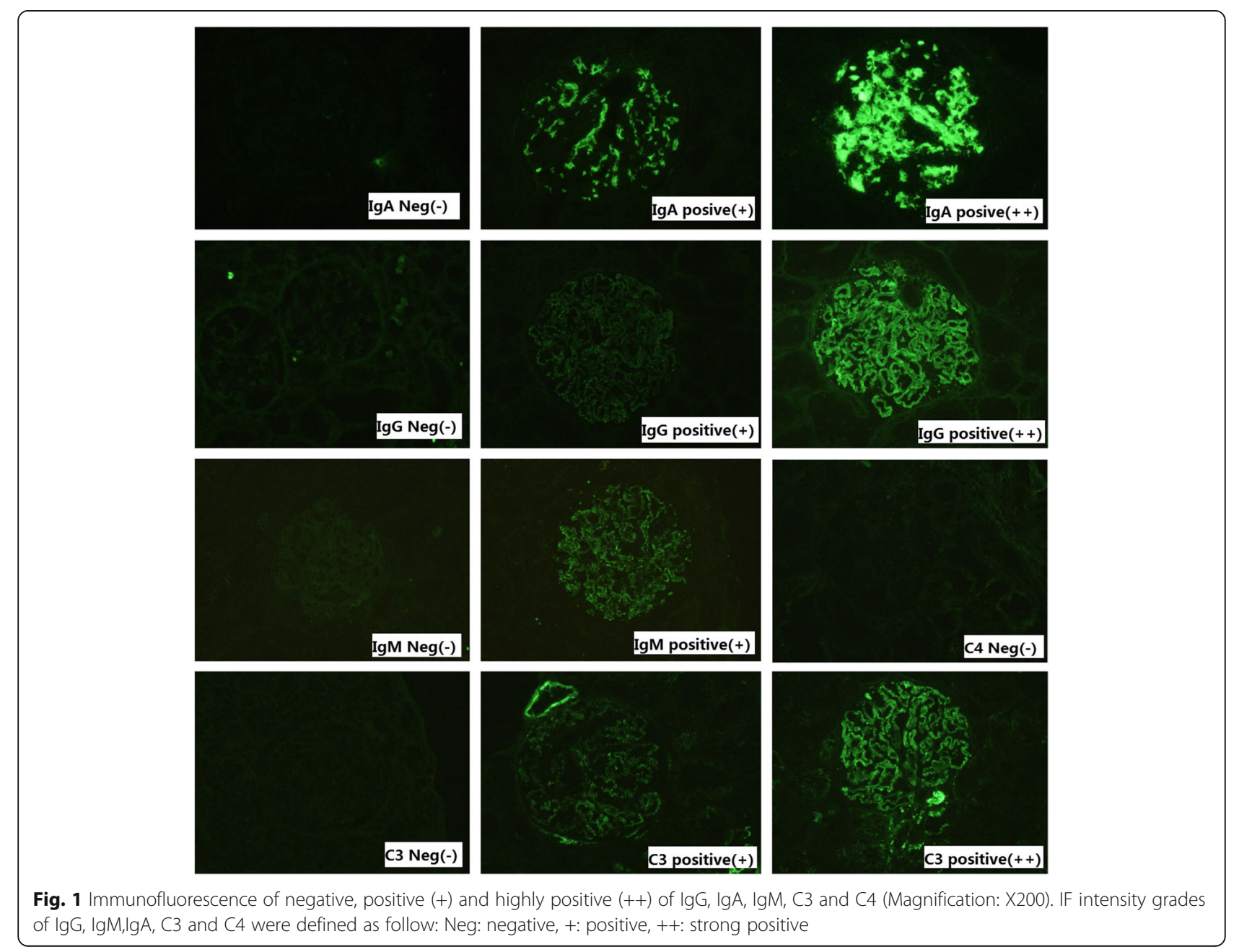


tested on Cobas analyzer (ROCHE). Estimated glomerular filtration rates (eGFR) (modified to Chinese population) were calculated [11].

\section{Groups divided}

According to the diagnosis of renal biopsies, two hundred and thirty-six patients were divided into three groups: IgAN group $(n=88), \mathrm{MN}$ group $(n=100), \mathrm{MCD}$ group $(n=48)$. IF intensity grades were defined as negative $(-)$, positive $(+)$, and strong positive $(++)$. Detailed characteristics of patients were shown in Table 1.

\section{Statistics}

Statistic analysis was performed on IBM SPSS Statistics software (version 19.0). Age and serum parameters were shown as means (95\% confidence interval, 95\% CI). Non-parametric tests (Mann-Whitney U test for comparison of two groups; Kruskal-Wallis $\mathrm{H}$ test for comparison of all Neg, positive $(+)$ and strong positive $(++)$ groups) and Chi-square test were used for the comparison among groups. $\mathrm{P}$ trend analysis was approximately evaluated by linear-by-linear association in Chi-square test. Correlation tests were used between serum immunoglobulin levels and renal function. $P$ value under 0.05 was considered as a cut-off value as statistically significant difference.

\section{Results}

\section{General characteristics of patients in IgAN, MN and MCD} groups

As described in Table 1, there were differences in IgA, IgG and C3 IF intensity grades among IgAN, MN and MCD groups $(p=9.82 \mathrm{E}-43,4.60 \mathrm{E}-39, \quad 7.45 \mathrm{E}-15$,respectively). Serum values of BUN, Crea, eGFR, IgG, IgA, IgM and C4 showed difference in three groups (BUN: $p=0.045$, Crea: $p=3.45 \mathrm{E}-5$, eGFR: $p=0.005$, IgG: $p=1.68 \mathrm{E}-14$, IgA: $p=9.14 \mathrm{E}-9, \operatorname{IgM}: p=0.014, \mathrm{C} 4: p=0.026)$.

\section{Correlation of IF intensity grades and kidney function}

Classification by IF intensity of immunoglobulin and complement as neg, positive $(+)$ and strong positive $(++)$ group, eGFR, BUN and Crea levels in each group were shown in Table 2. eGFR had the trend to decrease with enhanced IgA IF positive grades $(p=8.99 \mathrm{E}-4)$; Crea had trends to decrease with both enhanced IgA and IgG IF intensity grades $(p=2.06 \mathrm{E}-6,2.94 \mathrm{E}-5$, respectively $)$.

Table 1 General characteristics of patients in IgAN, MN and MCD groups

\begin{tabular}{|c|c|c|c|c|c|c|c|}
\hline \multicolumn{3}{|l|}{ Parameters } & IgAN & MN & MCD & Total & $P$ value \\
\hline \multicolumn{3}{|c|}{ Gender, n (female, \%) } & $88(34.1)$ & $100(41.0)$ & $48(37.5)$ & $236(100)$ & 0.621 \\
\hline \multicolumn{3}{|l|}{ age, year $(95 \% \mathrm{Cl})$} & $38.1(35.8-40.6)$ & $48.3(45.3-51.2)$ & $36.9(32.7-41.1)$ & - & $6.34 \mathrm{E}-6$ \\
\hline \multirow[t]{12}{*}{ IF intensity grade } & \multirow[t]{3}{*}{$\lg G$} & Neg & $71(80.7 \%)$ & $7(7.0 \%)$ & $48(100.0 \%)$ & $126(53.4 \%)$ & \multirow[t]{3}{*}{$4.60 \mathrm{E}-39$} \\
\hline & & + & 17(19.3\%) & $16(16.0 \%)$ & $0(0.0 \%)$ & $33(14.0 \%)$ & \\
\hline & & ++ & $0(0.0 \%)$ & $77(77.0 \%)$ & $0(0.0 \%)$ & $77(32.6 \%)$ & \\
\hline & \multirow[t]{2}{*}{$\lg M$} & Neg & $84(95.5 \%)$ & 99(99.0\%) & 47(97.9\%) & 230(97.5\%) & \multirow[t]{2}{*}{0.297} \\
\hline & & + & $4(4.5 \%)$ & $1(1.0 \%)$ & $1(2.1 \%)$ & $6(2.5 \%)$ & \\
\hline & \multirow[t]{3}{*}{$\lg A$} & Neg & $0(0.0 \%)$ & $91(91.0 \%)$ & 48(100\%) & 139(58.9\%) & \multirow[t]{3}{*}{$9.82 \mathrm{E}-43$} \\
\hline & & + & $32(36.4 \%)$ & $5(5.0 \%)$ & $0(0.0 \%)$ & $37(15.7 \%)$ & \\
\hline & & ++ & $56(63.6 \%)$ & $4(4.0 \%)$ & $0(0.0 \%)$ & $60(25.4 \%)$ & \\
\hline & \multirow[t]{3}{*}{ C3 } & Neg & $27(30.7 \%)$ & 39(39.0\%) & 48(100.0\%) & $114(48.3 \%)$ & \multirow[t]{3}{*}{$7.45 \mathrm{E}-15$} \\
\hline & & + & $50(56.8 \%)$ & $58(58.0 \%)$ & $0(0.0 \%)$ & $108(45.8 \%)$ & \\
\hline & & ++ & $11(12.5 \%)$ & $3(3.0 \%)$ & $0(0.0 \%)$ & $14(5.9)$ & \\
\hline & $\mathrm{C} 4$ & Neg & $88(100.0 \%)$ & $100(100.0 \%)$ & $48(100.0 \%)$ & $236(100.0 \%)$ & - \\
\hline \multirow[t]{8}{*}{ Serum values } & \multicolumn{2}{|c|}{ Urea, mmol/L } & $6.27(5.63-6.92)$ & $5.47(4.95-5.99)$ & $6.25(5.13-7.38)$ & - & 0.045 \\
\hline & \multicolumn{2}{|c|}{ Crea, $\mu \mathrm{mol} / \mathrm{L}$} & 111.6(100.1-123.2) & $76.1(71.1-81.0)$ & $81.7(75.6-87.7)$ & & $3.45 \mathrm{E}-5$ \\
\hline & \multicolumn{2}{|c|}{ eGFR, $\mathrm{ml} / \mathrm{min}$ per $1.73 \mathrm{~m}^{2}$} & $83.2(76.2-90.3)$ & 101.2(96.4-106.0) & 103.4(96.0-110.8) & & 0.005 \\
\hline & \multicolumn{2}{|c|}{$\mathrm{slgG}, \mathrm{mg} / \mathrm{dL}$} & 1015.4(952.6-1078.1) & $619.0(565.3-672.6)$ & $518.3(422.0-614.5)$ & & $1.68 \mathrm{E}-14$ \\
\hline & \multicolumn{2}{|c|}{$\mathrm{slgM}, \mathrm{mg} / \mathrm{dL}$} & 106.8(95.3-118.4) & 129.1(110.4-147.9) & 139.0(121.4-156.6) & & 0.014 \\
\hline & \multicolumn{2}{|c|}{$\mathrm{s} \lg \mathrm{A}, \mathrm{mg} / \mathrm{dL}$} & 308.0(285.9-330.1) & $221.1(203.5-238.8)$ & 211.7(185.4-237.9) & & $9.14 \mathrm{E}-9$ \\
\hline & \multicolumn{2}{|c|}{$\mathrm{sC} 3, \mathrm{mg} / \mathrm{dL}$} & 109.2(104.8-113.5) & 116.0(111.0-120.9) & $117.0(110.0-124.0)$ & & 0.124 \\
\hline & \multicolumn{2}{|c|}{$\mathrm{sC} 4, \mathrm{mg} / \mathrm{dL}$} & $25.8(24.3-27.3)$ & $29.9(27.8-32.0)$ & $28.1(25.0-31.1)$ & & 0.026 \\
\hline
\end{tabular}

Results were shown as mean $(95 \% \mathrm{Cl})$. eGFR was estimated by MDRD formula [1]. IF intensity grade of $\operatorname{lgG}, \lg M, \lg A, C 3$ and $C 4$ was defined as following: Neg: negative, +: positive, ++: strong positive 
Table 2 Comparison of kidney function among different IF grades of immunoglobulin and complement

\begin{tabular}{|c|c|c|c|c|c|c|c|c|}
\hline \multicolumn{2}{|c|}{ Parameters } & \multirow{2}{*}{$\begin{array}{l}\mathrm{n} \\
126\end{array}$} & \multirow{2}{*}{$\begin{array}{l}\text { eGFR, } \mathrm{ml} / \mathrm{min} \text { per } 1.73 \mathrm{~m}^{2} \\
89.7(84.1-95.4)\end{array}$} & \multirow{2}{*}{$\begin{array}{c}P_{\text {trend }} \\
0.054\end{array}$} & \multirow{2}{*}{$\begin{array}{l}\text { Urea, } \mathrm{mmol} / \mathrm{L} \\
6.33(5.72-6.94)\end{array}$} & \multirow{2}{*}{$\begin{array}{l}P_{\text {trend }} \\
0.167\end{array}$} & \multirow{2}{*}{$\begin{array}{l}\text { Crea, } \mu \mathrm{mol} / \mathrm{L} \\
100.7(92.0-109.5)\end{array}$} & \multirow{2}{*}{$\frac{P_{\text {trend }}}{2.94 \mathrm{E}-5}$} \\
\hline $\lg G$ & Neg & & & & & & & \\
\hline & + & 33 & $98.9(88.5-109.3)$ & & $5.42(4.32-6.51)$ & & 85.0(74.6-95.4) & \\
\hline & ++ & 77 & 101.7(96.5-107.0) & & $5.49(4.98-6.00)$ & & $76.0(70.7-81.3)$ & \\
\hline \multirow[t]{2}{*}{$\lg M$} & Neg & 230 & 95.2(91.4-99.0) & 0.359 & $5.93(5.52-6.33)$ & 0.326 & $90.0(84.6-95.4)$ & 0.133 \\
\hline & + & 6 & 83.6(49.2-117.9) & & $5.97(4.84-7.09)$ & & $107.7(67.2-148.2)$ & \\
\hline \multirow[t]{3}{*}{$\lg A$} & Neg & 139 & $102.1(98.1-106.1)$ & 8.99E-4 & $5.75(5.21-6.27)$ & 0.036 & 77.5(73.7-81.4) & $2.06 \mathrm{E}-6$ \\
\hline & + & 37 & $75.8(64.6-87.0)$ & & $6.34(5.55-7.13)$ & & 117.7(101.5-133.8) & \\
\hline & ++ & 60 & $90.1(81.8-98.5)$ & & $6.09(5.25-6.93)$ & & 103.6(89.1-118.2) & \\
\hline \multirow[t]{3}{*}{ C3 } & Neg & 114 & $99.4(94.2-104.7)$ & 0.080 & $5.89(5.29-6.49)$ & 0.269 & 85.3(78.7-92.0) & 0.195 \\
\hline & + & 108 & $90.3(84.4-96.2)$ & & $6.07(5.49-6.65)$ & & $96.1(87.2-104.9)$ & \\
\hline & ++ & 14 & $94.4(80.8-108.0)$ & & $5.18(4.14-6.22)$ & & $89.0(64.7-113.4)$ & \\
\hline C4 & Neg & 236 & $94.9(91.2-98.7)$ & - & $5.93(5.53-6.32)$ & - & $90.5(85.1-95.8)$ & - \\
\hline
\end{tabular}

Results were shown as mean(95\% Cl). eGFR was estimated by MDRD formula [1]

IF intensity grades of $\lg G, \lg M, \lg A, C 3$ and $C 4$ were defined as following: Neg: negative, +: positive, ++: strong positive

eGFR, BUN and Crea levels had no significantly changes among those groups.

Furthermore, we analyzed the correlation between eGFR, BUN, Crea levels and serum IgA, IgG levels. As shown in Fig. 2, in all subjects, serum IgA levels were inversely correlated with $\operatorname{eGFR}(r=-0.216, p=0.001)$ and correlated with Crea levels $(r=0.189, p=0.004)$; serum IgG and Crea showed no correlation which were discordance with inverse correltion of IgG IF grade and Crea $(r=0.058, p=0.379)$. An additional full parameters correlation analysis was shown as Additional file 1: Table S1.

In IgAN cases, not IgA but IgG was correlated to renal function indicators (serum IgG and eGFR: $r=-0.258$, $p=0.015$; serum IgA and Crea: $r=0.224, p=0.036$ ). In $\mathrm{MN}$ cases, only serum IgA was correlated with eGFR $(r=-0.234, p=0.020)$ (Additional file 1: Table S1).

The cases included in this study were almost at the early stage of kidney disease (CKD1 or CKD2), so the relationship between IF intensity and relevant serum level was not investigated in the patients above CKD3 stage $(\mathrm{eGFR}<60 \mathrm{ml} / \mathrm{min}$ per $1.73 \mathrm{~m} 2)$. The observation in whole CKD stages is very important. However, there exists a contradiction in clinical that is end-stage renal disease is a contraindication for renal biopsy. It is not easy to observe the IF intensity changes of tissue immune proteins when patient's renal function is very poor.

\section{Comparison of immunoprotein IF intensity grade and serum levels in total samples and in groups}

All immunoprotein IF grades and relevant serum levels were compared in all subjects. As shown in Fig. 3, in all subjects analysis, IgG serum level was inverse correlated with its IF intensity (i.e. serum IgG level decreased with enhanced IgG fluorescence intensity, $p=3.54 \mathrm{E}-5, p=$ 7.08E-6, respectively); C3 serum levels had significantly difference between Neg and positive $(+)$ group ( $p=$ 0.0003). IgA serum level was positive correlated with its IF grades (i.e. serum IgA level increased with enhanced IgA IF grades, Neg-(+): $p=0.0001 ;(+)-(++): p=0.022$; Neg- $(++): p=2.01 \mathrm{E}-10)$. From Fig. 3, though C3 serum levels had trend to decrease in the pace with C3 IF intensity grade $(\mathrm{Neg},+,++)$, statistical analysis showed no

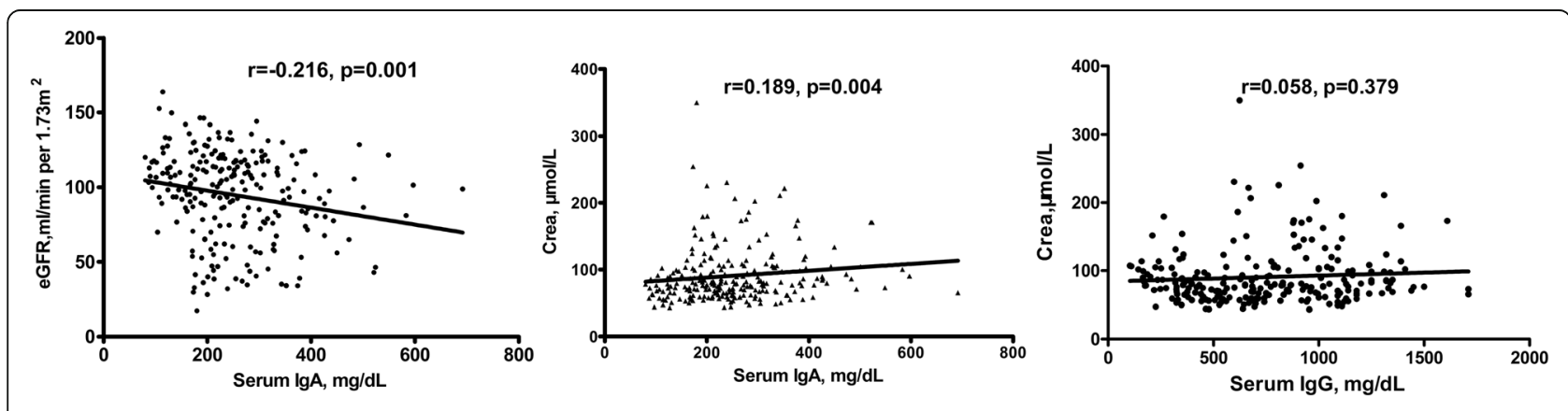

Fig. 2 Correlation of serum IgA and IgG level with eGFR and Crea levels 

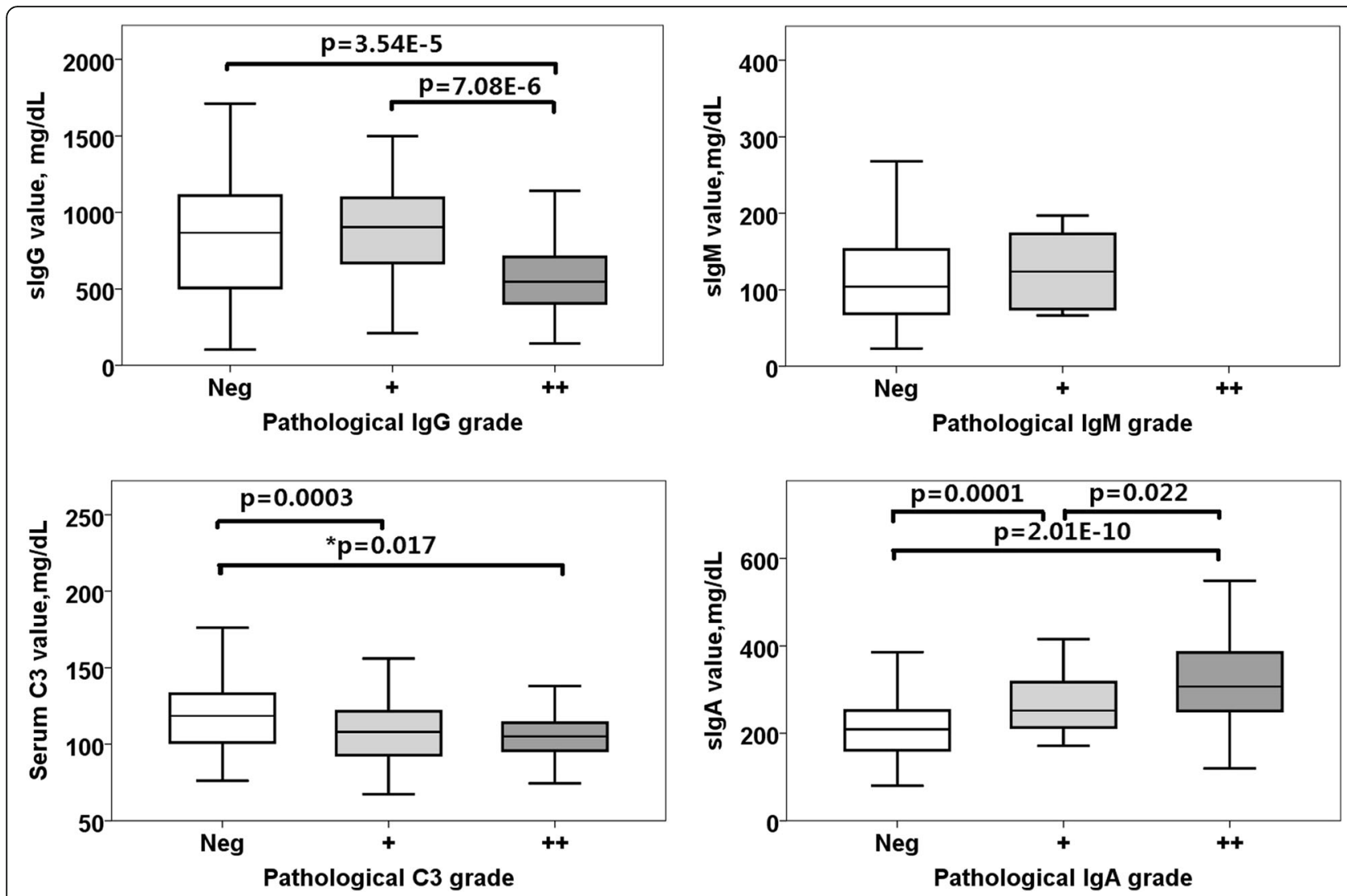

Fig. 3 Comparison of immunoprotein IF intensities and relevant serum levels. Parameters with $p$ or ${ }^{*} p$ value under 0.05 were not listed. Pathological C3 group (++) $(n=14)$, for matching comparison with C3 group (Neg) and group (+), 20\% samples were picked up randomly $(n=28$, respectively).* $p$ value were comparison of C3 Neg group( $\mathrm{n}=28)$ and C3 (++)group. IF intensity grades of IgG, IgM,lgA, C3 and C4 were defined as follow: Neg: negative, +: positive, ++: strong positive

significant difference $(p=0.067$ in $\mathrm{C} 3 \mathrm{Neg}$ and $\mathrm{C} 3++$ group, $p=0.929$ in $\mathrm{C} 3+$ and $\mathrm{C} 3++$ group). This contradict result may be caused by unbalanced sample numbers. As shown in Table 1, in 'Total” column, 114 samples were in C3 Neg. group, 108 samples were in C3 + group and only 14 samples were in $\mathrm{C} 3++$ group. Statistical difference may be assumed with more $\mathrm{C} 3++$ samples. For matching comparison among C3 groups, we analyzed again. 20\% samples from C3 Neg. group and C3 + group ( $n=28$, respectively) were randomly picked up, and then statistical significance appeared between C3 Neg. group and C3 ++ group ( $" p=0.017)$. We have added the result into Fig. 3, labeled as "p. C4 had all negative IF expression in all pathological groups, thus C4 data was not listed in Fig. 3).

We further explored all immunoprotein IF intensity grades and their serum values in IgAN, MN and MCD group (Fig. 4).

As shown in Fig. 4, the results showed that in IgAN subjects, there were statistical differences of serum C3 levels between its pathological Neg and positive $(+)$ group $(p=0.026)$, and serum IgA levels showed difference between IgA pathological positive $(+)$ and $(++)(p=0.007)$. In MN subjects, sIgG levels showed difference between IgG pathological If grade positive $(+)$ and $(++)(p=0.044)$; serum C3 levels showed difference between C3 pathological IF grade Neg and positive $(+)(p=0.005)$; and serum IgA levels showed difference between Neg and positive $(+)(p=0.040)$. In MCD subjects, most of the IF intensities were pathologically reported negative, so the analysis could not be done.

Moreover, based on the analysis of pathological group comparisons of IgAN and MN, we further did trend correlation analysis for investigating the relationships of IgG, IgA and C3 IF intensity grades and their serum concentrations and renal function in IgAN and MN (Tables 3, 4 and 5). To confirm the uptrend or downtrend of those serum parameter values, we did an additional correlation analysis as Additional file 1: Table S2.

In IgAN, eGFR showed serum IgA levels had significant differences among $\operatorname{groups}(p=0.007)$ and had increasing trend with enhanced its IF grades $\left(\mathrm{P}_{\text {trend }}=\right.$ 0.016)(Table 4). There were also difference between IgG group Neg and positive $(+)\left(p=0.005, \mathrm{P}_{\text {trend }}=0.007\right)$ in IgAN(Table 3,IgAN). 

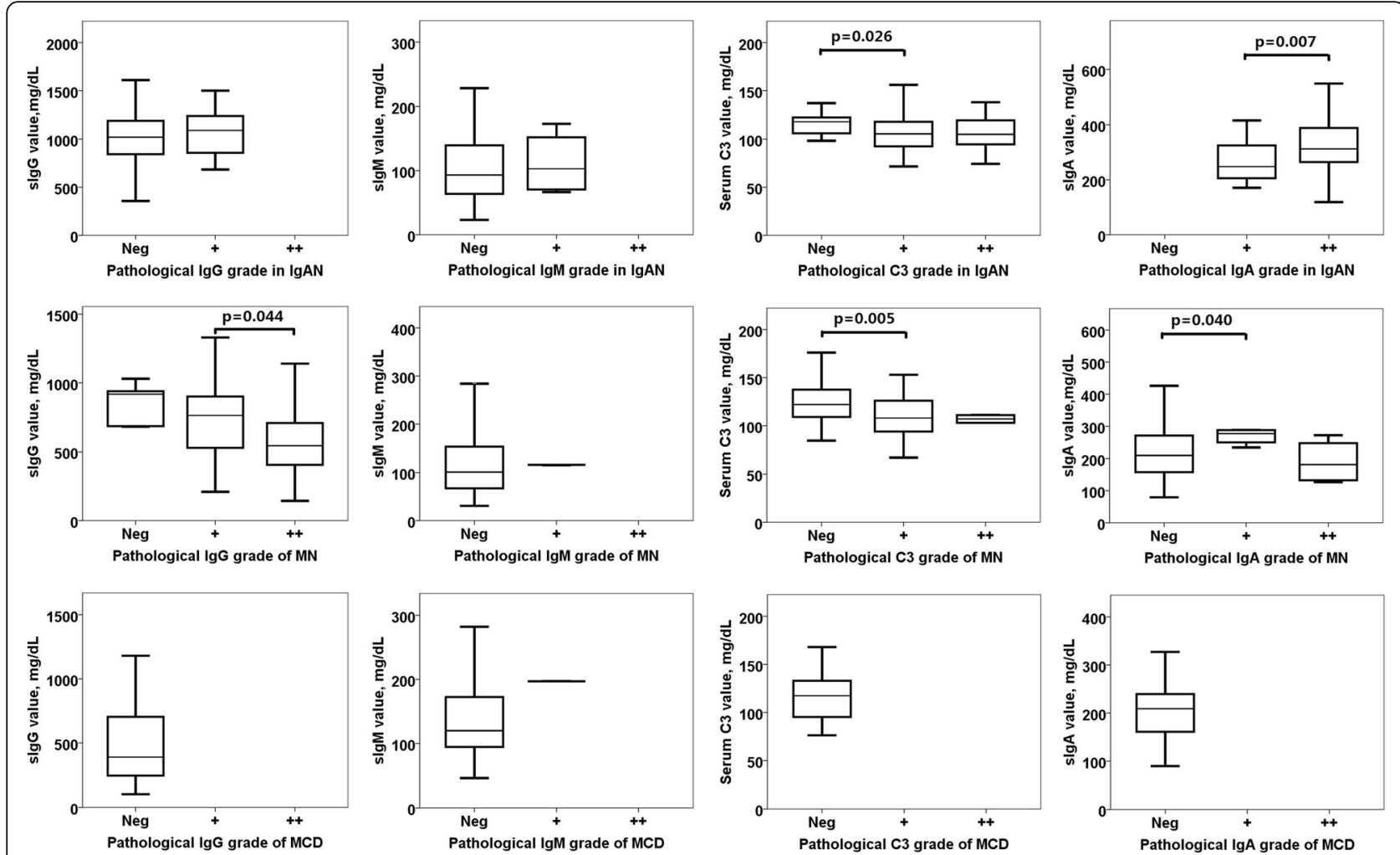

Fig. 4 Comparison of IF intensity grades and serum levels of immunoglobulin and complement in group of IgAN, MN and MCD. Parameters with $p$ value under 0.05 were not listed. IF intensity grades of IgG, IgM,IgA, C3 and C4 were defined as follow: Neg: negative, +: positive, ++: strong positive

Table 3 Comparison of IF intensity grades and serum levels of IgG between IgAN and MN

\begin{tabular}{|c|c|c|c|c|c|c|}
\hline & \multirow[t]{2}{*}{ Parameters } & \multicolumn{3}{|l|}{ lgG pathological grade } & \multirow[t]{2}{*}{$P$ value } & \multirow[t]{2}{*}{$P_{\text {trend }}$} \\
\hline & & Neg & + & ++ & & \\
\hline \multirow[t]{6}{*}{ IgAN } & $\mathrm{N}$ (female) & $71(26)$ & $17(4)$ & - & 0.234 & - \\
\hline & Age & 39.7(36.9-42.4) & $32.1(27.9-36.3)$ & - & 0.014 & 0.015 \\
\hline & Un & $6.52(5.74-7.29)$ & $5.24(4.58-5.91)$ & - & 0.129 & 0.120 \\
\hline & $\mathrm{Cr}$ & 117.3(103.4-131.1) & $88.1(76.3-99.9)$ & - & 0.117 & 0.048 \\
\hline & eGFR & $78.6(70.8-86.5)$ & 102.6(89.1-116.1) & - & 0.005 & 0.007 \\
\hline & $\operatorname{slg} G$ & 1013.2(942.7-1083.7) & 1024.4(871.7-1177.1) & - & 0.779 & 0.889 \\
\hline \multirow[t]{6}{*}{ MN } & $\mathrm{N}($ female) & $7(3)$ & $16(8)$ & $77(30)$ & 0.712 & - \\
\hline & Age & $51.0(42.2-59.8)$ & $51.3(42.0-60.6)$ & $47.4(43.9-50.8)$ & 0.554 & 0.301 \\
\hline & Un & $4.90(3.77-6.03)$ & $5.68(3.22-8.14)$ & $5.50(4.99-6.02)$ & 0.555 & 0.703 \\
\hline & $\mathrm{Cr}$ & $64.2(53.8-74.5)$ & $82.8(62.7-103.0)$ & $76.0(70.6-81.3)$ & 0.383 & 0.601 \\
\hline & eGFR & 108.8(96.2-121.5) & $94.0(75.5-112.5)$ & 101.6(96.4-106.9) & 0.667 & 0.950 \\
\hline & slgG & 777.9(529.1-1026.6) & 727.7(561.9-893.5) & $582.9(525.0-640.7)$ & 0.034 & 0.014 \\
\hline
\end{tabular}

Pathological grades were IF intensity grades of $\lg , \lg M, \lg A, C 3$ and $C 4$ were defined as following: Neg: negative, +: positive, ++: strong positive. $P$ value showed difference among Neg, positive $(+)$ and $(++)$ (analyzed by nonparametric tests-Kruskal-Wallis $\mathrm{H}$ test). $\mathrm{P}_{\text {trend }}$ showed serum immunoglobulin or compliment trends with their enhanced Pathological grade. $\mathrm{P}$ and $\mathrm{P}_{\text {trend }}$ values under 0.05 were considered as cut-off values as statistically significant differences 
Table 4 Comparison of IF intensity grades and serum levels of IgA between IgAN and MN

\begin{tabular}{|c|c|c|c|c|c|c|}
\hline & \multirow[t]{2}{*}{ Parameters } & \multicolumn{3}{|c|}{ IgA pathological grade } & \multirow[t]{2}{*}{$P$ value } & \multirow[t]{2}{*}{$P_{\text {trend }}$} \\
\hline & & Neg & + & ++ & & \\
\hline \multirow[t]{6}{*}{$\operatorname{IgAN}$} & $\mathrm{N}$ (female) & - & $32(9)$ & $56(21)$ & 0.257 & - \\
\hline & Age & - & $41.8(36.9-46.6)$ & $36.2(33.5-38.8)$ & 0.039 & 0.028 \\
\hline & Un & - & $6.46(5.57-7.34)$ & $6.17(5.27-7.06)$ & 0.288 & 0.669 \\
\hline & $\mathrm{Cr}$ & - & $120.5(102.7-138.2)$ & 106.6(91.3-121.9) & 0.082 & 0.251 \\
\hline & eGFR & - & $74.6(62.8-86.5)$ & $88.2(79.5-96.9)$ & 0.051 & 0.065 \\
\hline & $s \lg A$ & - & $272.5(240.0-304.9)$ & $328.3(299.5-357.1)$ & 0.007 & 0.016 \\
\hline \multirow[t]{6}{*}{ MN } & $\mathrm{N}($ female) & $91(37)$ & $5(3)$ & $4(1)$ & 0.556 & - \\
\hline & Age & $48.7(45.4-51.9)$ & $42.6(29.3-55.9)$ & $45.3(34.1-56.5)$ & 0.462 & 0.421 \\
\hline & Un & $5.50(4.93-6.08)$ & $5.61(3.27-7.96)$ & $5.05(3.10-7.00)$ & 0.815 & 0.822 \\
\hline & $\mathrm{Cr}$ & $75.4(70.4-80.5)$ & $99.9(46.4-153.5)$ & $62.7(40.4-85.0)$ & 0.212 & 0.908 \\
\hline & eGFR & 101.2(96.3-106.2) & 83.5(33.0-134.0) & 117.5(99.9-135.0) & 0.183 & 0.693 \\
\hline & $s \lg A$ & $218.9(200.1-237.7)$ & $285.0(218.0-352.0)$ & 190.5(79.4-301.6) & 0.092 & 0.847 \\
\hline
\end{tabular}

Pathological grades were IF intensity grades of IgG, IgM,IgA, C3 and C4 were defined as following: Neg: negative, $+:$ positive, $++:$ strong positive. $P$ value showed difference among Neg, positive $(+)$ and $(++)$ (analyzed by nonparametric tests-Kruskal-Wallis $\mathrm{H}$ test). $\mathrm{P}_{\text {trend }}$ showed serum immunoglobulin or compliment trends with their enhanced Pathological grade. $P$ and $P_{\text {trend }}$ values under 0.05 were considered as cut-off values as statistically significant differences

In $\mathrm{MN}$, serum IgG levels had significant differences among IF groups $(p=0.034)$ and was decreasing trend with its enhanced IF grades $\left(P_{\text {trend }}=0.014\right)($ Table $3, M N)$. Serum C3 concentrations also were found distinctive among IF groups $(p=0.016)$ and had in inverse correlation with its enhanced IF grades $\left(\mathrm{P}_{\text {trend }}=0.004\right)($ Table $5, \mathrm{MN})$.

\section{Discussion}

The morbidity of IgAN, MN and MCD has increased yearly, and IgAN is still dominant in primary glomerulonephritis [1]. Pathological results play decisive role in the diagnosis of nephropathy, by which kidney diseases are classified. Primary IgA nephropathy is characterized with IgA-dominant deposition in the glomerulus [12]; in $\mathrm{MN}$, the pathological characteristics is IgG-dominant deposits [13] mostly with C3 deposits [14], and IgM or IgA has also been reported in $\mathrm{MN}[15,16]$; normally, less immonoprotein depositions are found in the patients with MCD [17]; few researches find that IgA, IgM, $\mathrm{C} 3$ or $\mathrm{C} 1 \mathrm{q}$ immune complex could also exist in $\mathrm{MCD}$ [18-20].

Hence, the formation and the deposition of immunoprotein complexes are closely related with pathogenesis of primary glomerular nephritis. In our study, on

Table 5 Comparison of IF intensity grades and serum levels of C3 between IgAN and MN

\begin{tabular}{|c|c|c|c|c|c|c|}
\hline & \multirow[t]{2}{*}{ Parameters } & \multicolumn{3}{|c|}{ C3 pathological grade } & \multirow[t]{2}{*}{$P$ value } & \multirow[t]{2}{*}{$P_{\text {trend }}$} \\
\hline & & Neg & + & ++ & & \\
\hline \multirow[t]{6}{*}{ IgAN } & $\mathrm{N}($ female) & $27(8)$ & $50(19)$ & $11(3)$ & 0.668 & - \\
\hline & Age & $37.1(32.6-41.6)$ & $38.4(34.9-42.0)$ & 39.9(35.7-44.2) & 0.615 & 0.470 \\
\hline & Un & 6.34(4.98-7.71) & $6.40(5.54-7.26)$ & $5.51(4.25-6.77)$ & 0.510 & 0.567 \\
\hline & $\mathrm{Cr}$ & 109.2(87.6-130.7) & 116.8(100.8-132.7) & $94.4(63.2-125.6)$ & 0.346 & 0.703 \\
\hline & eGFR & $86.9(73.5-100.4)$ & $78.7(69.1-88.3)$ & $94.9(77.6-112.2)$ & 0.326 & 0.884 \\
\hline & $\mathrm{sC3}$ & 115.6(108.6-122.7) & $106.2(100.1-112.3)$ & 106.9(93.0-120.8) & 0.079 & 0.102 \\
\hline \multirow[t]{6}{*}{ MN } & $\mathrm{N}($ female) & $39(17)$ & $58(22)$ & $3(2)$ & 0.562 & - \\
\hline & Age & $49.5(45.1-53.9)$ & $47.0(42.7-51.2)$ & $57.3(42.2-72.5)$ & 0.443 & 0.781 \\
\hline & Un & $5.11(4.52-5.70)$ & $5.80(4.99-6.62)$ & $3.95(1.96-5.94)$ & 0.252 & 0.514 \\
\hline & $\mathrm{Cr}$ & $73.4(65.0-81.7)$ & 78.3(71.5-85.0) & 72.4(35.6-109.2) & 0.323 & 0.525 \\
\hline & eGFR & 103.2(94.9-111.4) & 100.1(93.7-106.5) & 83.6(0-179.5) & 0.410 & 0.425 \\
\hline & $\mathrm{sC} 3$ & $125.0(116.9-133.2)$ & 110.1(104.0-116.2) & 107.0(56.2-157.8) & 0.016 & 0.004 \\
\hline
\end{tabular}

Pathological grades were IF intensity grades of $\lg , \lg M, \lg A, C 3$ and $C 4$ were defined as following: Neg: negative, $+:$ positive, $++:$ strong positive. $P$ value showed difference among Neg, positive $(+)$ and $(++)$ (analyzed by nonparametric tests-Kruskal-Wallis $\mathrm{H}$ test). $\mathrm{P}_{\text {trend }}$ showed serum immunoglobulin or compliment trends with their enhanced Pathological grade. $P$ and $P_{\text {trend }}$ values under 0.05 were considered as cut-off values as statistically significant differences 
analysis of all subjects, we found serum IgG and C3 concentrations trended to decrease with their enhanced IF grades, while serum IgA concentration elevated with the enhanced IgA IF intensity. Our analysis showed that in MN patients, serum IgG level declined with the enhanced IgG IF intensity. Interestingly, unlike changes of IgG, IgA in pathological level or serum level showed very different changes. Serum IgA value increased with the enhanced renal tissue IgA IF intensity. This may be related to the diversity mechanism of immune complex formation in IgAN and MN. Until now, the pathogenesis of IgAN remains unclear. Some scholars found that in the patients with IgAN, besides renal IgA complexes, there also was IgA complexes accumulating in the tissue of liver [12] or skin [21]. In the physiologic state, IgA can be synthesized in many tissues. IgA is composed of two subclasses as IgA1 and IgA2. Approximately $85 \%$ IgA in serum is IgA1. IgA1 complexes are the main deposition in IgAN [22]. Therefore, the immune dysfunction in IgAN is involved not only in kidney but also in multiple systems and tissues. In our study, serum IgA (mainly IgA1) remaining high levels, that might be caused by the abnormal activation of immune system involved in multiple tissues, was correlated with the pathological IgA IF intensity grades. Multiple systems and tissues are involved in the changes. Some other observations also had conflict on initiating pathogenesis of IgAN [23, 24]. It is still unable to affirmed that which one is the initial tissue, kidney or others organs. Our analysis also indicated that $\mathrm{C} 3$ had synergistic effect in IgAN, which was correlated with serum IgA level. There were some reports found that IgA/C3 index, to some extent, was valuable in the diagnosis of IgAN $[4,6]$. In MN patients, both serum IgG and C3 levels are reversely correlated with their respective IF intensity grades. The pathological characteristic of $\mathrm{MN}$ is the formation of immune complexes (mainly IgG and C3 complexes) deposit in glomerular capillary wall in situ [4]. Our analysis indicates that the IgG deposition might not source from circulating immune complexes: the formation of high levels of IgG-complexes had limited in nephridial tissue and less in other systems. In addition, the activation of complement system is also one of the factors of renal impairment. However, our results indicated that the pathogenesis of MN, unlike that of IgAN, might be an incipient and restrictive immune disease in kidney.

eGFR is a common indicator in clinic for estimation of renal function using gender, age and serum creatinine, which was modified by Ma, Y.C [11] for Chinese population. Our result showed that, there were decreased eGFR and elevated serum creatinine with positive IgA IF level, which was corresponding to trends of serum IgA level with eGFR and serum creatinine. The result indicated that the formation of IgA complexes aggravated dysfunction of kidney. Our result also showed that IgG IF intensity grade was correlated with serum creatinine, while irrelevant with eGFR. Since eGFR is calculated with several index (gender, age and serum creatinine), the factors of gender, age also affect calculation of eGFR; moreover, significant difference may be found with sample enlarged. In separate disease analysis, interestingly, we found that in IgAN patients (characteristic as positive IgA deposition), lower eGFR appeared in IgG negative patients than in IgG positive patients. As reported in some researches $[8,10,25]$, in IgA nephropathy patients, IgG deposition showed at different degrees. In Nasri $\mathrm{H}^{\prime}$ reports, no significant association of IgG deposition with serum creatinine [10], and only C3 deposits had a significant correlation with serum creatinine [9]. In Wada Y's study [8], proteinuria was greater in the both IgA-IgG positive group than the IgA positive group in IgAN patients and conclude that mesangial IgG deposition is associated with more severe clinical features in IgAN patients. The conflict of results of IgG and serum creatinine(and eFGR) may caused by following reasons: i. the subjects: As illustrated, the subjects included in this study were almost at the early stage of CKD1 or CKD2, and end-stage renal disease is a contraindication for renal biopsy. Besides, age might be another cause for age of IgG Neg group was 39.7(36.9-42.4) and age of positive (+) group was 32.1(27.9-36.3) in IgAN. ii. IgG positive grade: of all subjects in this study, we found no IgG strong positive $(++)$ cases, while in Nasri H's [10] and Wada Y's [8] studies, there were cases involved with IgG IF strong positive grades. But from our present data, tissue IgG IF hardly showed strong positive in IgAN patients, which might caused by population diversities or IgG might played different role in pathogenesis and development of IgAN. The study implied that the positive rate of IgA and IgG complexes was relevant with renal function, in addition $\mathrm{C} 3$ might play roles in pathogenesis of primary glomerular nephritis, but was irrelevant with renal dysfunction.

Serum urea nitrogen and creatinine are most common indicators for estimation of renal function. In this study on IgG IF intensity grade, we found that serum creatinine decreased with increased IgG positive IF grades; while serum creatinine evaluated with increased IgA positive IF grades; same tendency was found in serological level. Serum IgG level was found uncorrelated with eGFR, nor serum urea nitrogen or creatinine. Zhang J, et al. [4] reported that serum IgA/C3 index had predictive effects on IgAN involvement. Our result showed that IgA level (from both serological and pathological levels) was closely related with renal function, and might affect prognosis of kidney. Otherwise, increased IgG level shows none of similar relationships in $\mathrm{MN}$ patients. 
Medical diagnosis with artificial intelligence (AI) has been developed recent years. With BIG Data analysis (trend and risk analysis based on patients records and treatments), there are preliminary models set up for evaluation the risks, processes or diagnosis of disease $[26,27]$. There are some diagnostic models that have now been set up and trained for differentiation of diseases based on 'Big Data', such as diagnosis of skin cancer by classification of skin lesions using deep convolutional neural networks [28], Big data analytics has been used in many fields in medicine, mostly in the health care of the illness or the health $[29,30]$. As the golden standard for diagnosis of primary glomerular nephritis, histopatholgical examination is a invasive method including several steps of renal biopsy, sample collection, staining, and reading. However renal biopsy has limitations which are as follow: a. patients with the contraindication; b. case with medical disputes; c. adaptability and understanding of patients and their families. Our research cross contrasts several immunoprotein IF intensities and relevant serum levels in three kinds of primary glomerular nephritis, and finally acquired helpful results for understanding the relationships between pathological presentation and serological presentation of immunoproteins in kidney diseases. Furthermore, this pilot study is offering a possible method for the analysis of combination of pathology and serology.

\section{Conclusion}

Our study showed that the changes of immunoprotein especially IgA and IgG, indicate the different pathogenesis of IgAN and MN, furthermore pathologically or serologically increased IgA level is closely related with renal function.

The limitation of this study is the sample numbers. Each group needs to be enlarged for more convince results. And some new renal disease markers, such as cystatin $\mathrm{C}$ and lipoprotein phospholipase $\mathrm{A} 2$, are not included.

\section{Additional file}

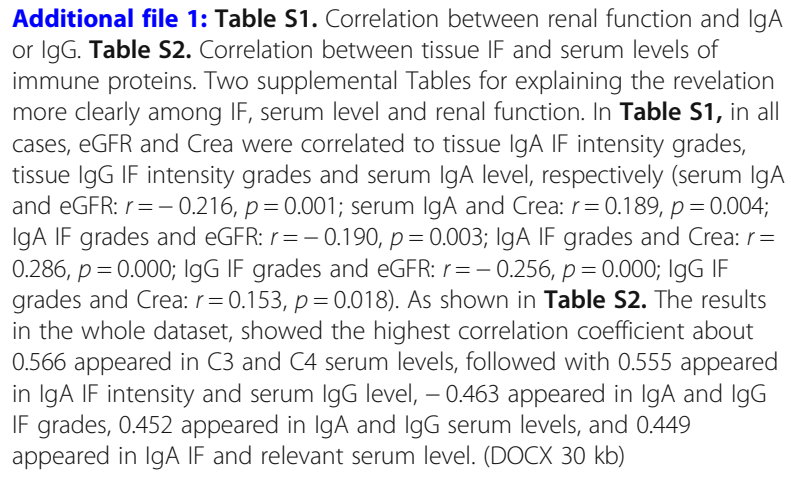

Additional file 1: Table S1. Correlation between renal function and lgA or IgG. Table S2. Correlation between tissue IF and serum levels of immune proteins. Two supplemental Tables for explaining the revelation more clearly among IF, serum level and renal function. In Table S1, in all cases, eGFR and Crea were correlated to tissue IgA IF intensity grades, tissue IgG IF intensity grades and serum IgA level, respectively (serum IgA and eGFR: $r=-0.216, p=0.001$; serum IgA and Crea: $r=0.189, p=0.004$; IgA IF grades and eGFR: $r=-0.190, p=0.003$; IgA IF grades and Crea: $r=$ $0.286, p=0.000$; lgG IF grades and eGFR: $r=-0.256, p=0.000$; $\lg \mathrm{IF}$ grades and Crea: $r=0.153, p=0.018$ ). As shown in Table S2. The results in the whole dataset, showed the highest correlation coefficient about 0.566 appeared in C3 and C4 serum levels, followed with 0.555 appeared in IgA IF intensity and serum IgG level, -0.463 appeared in IgA and IgG IF grades, 0.452 appeared in IgA and IgG serum levels, and 0.449 appeared in IgA IF and relevant serum level. (DOCX $30 \mathrm{~kb}$ )

\section{Abbreviations}

BUN: Urea nitrogen; C3: Complement C3; C4: Complement C4;

Crea: Creatinine; eGFR: estimated Glomerular Filtration Rate;

IF: Immunofluorescence; IgA: Immunoglobulin A; IgAN: Immunoglobulin A nephropathy; IgG: Immunoglobulin G; IgM: Immunoglobulin M; MCD: Minimal-change disease; MN: Membranous nephropathy

\section{Acknowledgements}

The authors would like to thank all staff in the Department of Clinical Biochemistry and Testing Center of Health Management Institute at the Chinese PLA General Hospital for their support.

\section{Funding}

This study was supported by Young science foundation of National Natural Science Foundation of China (81401719) and Chinese national 863 project (2014AA022304).

\section{Availability of data and materials}

Materials described in the manuscript, including all relevant raw data, will be freely available to any scientist wishing to use them for non-commercial purposes, without breaching participant confidentiality.

\section{Authors' contributions}

YW designed and reviewed this study. JD wrote the main manuscript. TH P collected the samples and data. JG and XW J performed the data analysis. GT $Y$ reviewed the main manuscript. All authors read and approved the manuscript.

\section{Ethics approval and consent to participate}

Ethics approval: This retrospective cohort study has been approved by the Medical Ethics Committee of Chinese PLA General Hospital, conformed to the principles outlined in the declaration of Helsinki and in accordance with the approved guidelines.

Ethics, consent and permissions: Informed consent to participate of this study has obtained from all participants.

\section{Consent for publication}

Informed consent to publish of this study has obtained from all participants.

\section{Competing interests}

The authors declare that they have no competing interests.

\section{Publisher's Note}

Springer Nature remains neutral with regard to jurisdictional claims in published maps and institutional affiliations.

\section{Author details}

${ }^{1}$ Department of Clinical Biochemistry, Medical Laboratory Center, State Key Laboratory of Kidney Disease, Chinese PLA General Hospital, Beijing 100853, China. ${ }^{2}$ Testing Center of Health Management Institute, Chinese PLA General Hospital, Beijing 100853, China. ${ }^{3}$ Department of Nephrology, Chinese PLA Institute of Nephrology, State Key Laboratory of Kidney Diseases, National Clinical Research Center for Kidney Diseases, Medical Institution Conducting Clinical Trials, Chinese PLA General Hospital, Beijing, China.

Received: 7 February 2018 Accepted: 7 June 2018

Published online: 20 June 2018

References

1. Xu X, et al. Analysis of 4931 renal biopsy data in Central China from 1994 to 2014. Ren Fail. 2016;38(7):1021-30.

2. Chu F, Chen G, Liu Y. Pathological patterns of primary nephrotic syndrome in Central China: a retrospective study of 627 cases. Ren Fail. 2014;36(4):514-9.

3. Yamada $\mathrm{N}$, et al. Relationship between immunoglobulin deposition and early lesions of progressive Glomerulonephropathy in young common marmosets. Vet Pathol. 2018:55(1):173-6.

4. Zhang J, et al. Serum immunoglobulin a/C3 ratio predicts progression of immunoglobulin a nephropathy. Nephrology (Carlton). 2013;18(2):125-31.

5. Yang $X$, et al. Decreased serum C3 levels in immunoglobulin a (IgA) nephropathy with chronic kidney disease: a propensity score matching study. Med Sci Monit. 2017;23:673-81. 
6. Mizerska-Wasiak M, et al. Relationship between serum IgA/C3 ratio and severity of histological lesions using the Oxford classification in children with IgA nephropathy. Pediatr Nephrol. 2015;30(7):1113-20.

7. Gao J, et al. A novel differential diagnostic model based on multiple biological parameters for immunoglobulin a nephropathy. BMC Med Inform Decis Mak. 2012;12:58.

8. Wada Y, et al. Clinical significance of IgG deposition in the glomerular mesangial area in patients with IgA nephropathy. Clin Exp Nephrol. 2013; 17(1):73-82

9. Nasri $\mathrm{H}$, et al. Correlation of immunostaining findings with demographic data and variables of Oxford classification in IgA nephropathy. J Nephropathol. 2013;2(3):190-5.

10. Nasri H. IgG deposition in IgA nephropathy patients. J Renal Inj Prev. 2013; 2(1):11-3.

11. Ma YC, et al. Modified glomerular filtration rate estimating equation for Chinese patients with chronic kidney disease. J Am Soc Nephrol. 2006; 17(10):2937-44.

12. Lai KN. Pathogenesis of IgA nephropathy. Nat Rev Nephrol. 2012;8(5):275-83.

13. Sinico RA, et al. Immunology of membranous nephropathy: from animal models to humans. Clin Exp Immunol. 2016;183(2):157-65.

14. Niel O, et al. C3 nephritic factor can be associated with membranous glomerulonephritis. Pediatr Nephrol. 2015;30(2):353-5.

15. Kobayashi M, et al. Membranous nephropathy with solitary immunoglobulin a deposition. Intern Med. 2015;54(9):1081-4.

16. Iwafuchi $Y$, et al. Schnitzler syndrome complicated by membranous nephropathy. Clin Nephrol. 2012;78(6):497-500.

17. Floege J, Amann K. Primary glomerulonephritides. Lancet. 2016;387(10032): 2036-48.

18. Pardo $V$, et al. The clinical significance of mesangial IgM deposits and mesangial hypercellularity in minimal change nephrotic syndrome. Am J Kidney Dis. 1984;3(4):264-9.

19. Niu HX, et al. Clinicopathological features, diagnosis, and treatment of IgA nephropathy with minimal change disease related to exposure to mercurycontaining cosmetics: a case report. Clin Nephrol. 2017;87(4):196-201.

20. Herlitz LC, et al. IgA nephropathy with minimal change disease. Clin J Am Soc Nephrol. 2014;9(6):1033-9.

21. Thompson AJ, et al. Vascular lgA deposits in clinically normal skin of patients with renal disease. Pathology. 1980;12(3):407-13.

22. Novak J, et al. Aberrant glycosylation of IgA1 and anti-glycan antibodies in IgA nephropathy: role of mucosal immune system. Adv Otorhinolaryngol. 2011;72:60-3.

23. Al Hussain T, et al. Pathophysiology of IgA nephropathy. Adv Anat Pathol. 2017;24(1):56-62

24. Suzuki $H$, et al. The pathophysiology of IgA nephropathy. J Am Soc Nephrol. 2011;22(10):1795-803.

25. Maixnerova D, et al. Markers for the progression of IgA nephropathy. J Nephrol. 2016;29(4):535-41.

26. Andreu-Perez J, et al. Big data for health. IEEE J Biomed Health Inform. 2015; 19(4):1193-208.

27. Viceconti M, Hunter P, Hose R. Big data, big knowledge: big data for personalized healthcare. IEEE J Biomed Health Inform. 2015;19(4):1209-15.

28. Esteva A, et al. Dermatologist-level classification of skin cancer with deep neural networks. Nature. 2017;542(7639):115-8.

29. Rumsfeld JS, Joynt KE, Maddox TM. Big data analytics to improve cardiovascular care: promise and challenges. Nat Rev Cardiol. 2016;13(6):350-9.

30. Tan SS, Gao G, Koch S. Big data and analytics in healthcare. Methods Inf Med. 2015;54(6):546-7.

\section{Ready to submit your research? Choose BMC and benefit from:}

- fast, convenient online submission

- thorough peer review by experienced researchers in your field

- rapid publication on acceptance

- support for research data, including large and complex data types

- gold Open Access which fosters wider collaboration and increased citations

- maximum visibility for your research: over $100 \mathrm{M}$ website views per year

At BMC, research is always in progress.

Learn more biomedcentral.com/submissions 